\title{
Social Isolation and Loneliness of Older Adults in Times of the COVID-19 Pandemic: Can Use of Online Social Media Sites and Video Chats Assist in Mitigating Social Isolation and Loneliness?
}

\author{
André Hajek Hans-Helmut König \\ Department of Health Economics and Health Services Research, University Medical Center Hamburg-Eppendorf, \\ Hamburg, Germany
}

\section{Keywords}

Social isolation - Social media use - Social exclusion .

Loneliness · Video call · Internet use · COVID-19.

SARS-CoV-2; Coronavirus

\begin{abstract}
The COVID-19 pandemic is a serious global burden. Epidemiological data suggest that the severity of COVID-19, in particular its case fatality rate, rises strongly with age. It is possible that neither a vaccine nor an effective treatment will be available for $>1$ year. Thus, it may be necessary for older adults to protect themselves by avoiding direct social contact and practicing social distancing for a rather long period of time. This may result in loneliness and social isolation because, for example, grandchildren cannot visit their grandparents. In turn, both loneliness and social isolation can have serious deleterious consequences (e.g., in terms of morbidity and mortality). Thus, the question arises: are there ways to mitigate loneliness and social isolation? One way to stay in contact is to use online social media such as Facebook or using video calling software such as Skype. However, there are very few studies examining whether the use
\end{abstract}

karger@karger.com

(c) 2020 S. Karger AG, Basel

www.karger.com/ger

Karger! of online social media or video chats are associated with loneliness and social isolation in older adults. We sum up some preliminary findings and make a call for further research on the link between online social media use/video chat and loneliness, as well as social isolation, in older adults.

(c) 2020 S. Karger AG, Basel

\section{Introduction}

The COVID-19 pandemic has already changed the world and will continue to do so. While the economic and social consequences are not completely predictable, there is little doubt that it will have an effect on many areas of life - at least in the midterm.

It is widely acknowledged that the severity of COVID-19, in particular its case fatality rate, increases considerably with age. One possible scenario is that older adults will be forced to stay home for extended periods of time to avoid physical contact with other people (e.g., when buying food) in order to avoid infection with the coronavirus. This can affect mental health and well-being among individuals in old age $[1,2]$. Ultimately, this can also lead 
to feelings of social isolation (feeling that one does not belong to the society) and loneliness (perceived discrepancy between actual and desired social relationships). This has been found to be the case, for example, when grandchildren cannot have direct contact with their grandparents. Loneliness and social isolation are correlated, but they are distinct constructs and differ in their predictors. While individuals can perceive themselves as socially isolated, they may not feel lonely at the same time and vice versa. Nevertheless, both social isolation and loneliness should be avoided because they can have important negative consequences for morbidity and mortality.

\section{Main Text}

One possible solution to cope with this situation and stay in contact with others is to use video chats (e.g., Skype) or online social media (e.g., Facebook). These solutions may at least temporarily substitute physical contact (e.g., with friends, acquaintances, children, or grandchildren) in times of the COVID-19 pandemic. It appears plausible that these possibilities can reduce feelings of social isolation or loneliness (e.g., when seeing their grandchildren on their monitor or smartphone). Moreover, older individuals using video chats or online social media sites to stay in contact with family and friends may think that they are better off than when they did not use such technical possibilities during self-isolation (intraindividual comparison). Thus, they may highly appreciate such technical solutions to overcome feelings of isolation and loneliness. Furthermore, they may compare themselves with other individuals who are not able to participate (e.g., for health reasons) in video chats or online social media sites (interindividual comparisons) and may realize that they are better off than these individuals. Both intraindividual and interindividual comparisons could lead to decreased feelings of social isolation or feelings of loneliness.

To date, very few studies have investigated the association between the use of video chats or online social media and social isolation or loneliness among older adults. We first summarize these preliminary findings before making a call for further research on the association between online social media use/video chat and social isolation among older adults. It is worth noting that we focused on nationally representative samples to present findings which are generalizable to the entire (community-dwelling) population of older adults in the spe- cific countries. We included studies focusing on individuals in their second half of life (i.e., 40 years and over [3]).

Based on nationally representative samples, only 3 studies have investigated the link between online social network sites and loneliness or social isolation among older adults. While a first study focused on various outcomes (i.e., social connectedness and social isolation) [4], a second study focused on loneliness [5], and a third study focused on social isolation as the outcome measure [6].

Based on data from the nationally representative Health and Retirement Study $(n=1,620$ individuals aged 50 years and over in the United States), the first study [4] showed a link between use of social network sites and increased feelings of connectedness (an aspect of loneliness), whereas the use of these sites was not associated with feelings of social isolation. Based on a sample of community-dwelling individuals aged 60 years and over ( $n=626)$ in the Netherlands, the second study revealed that the use of these sites was neither associated with general loneliness nor with social or emotional loneliness [5]. The third study [6] examined the link between social network sites and social isolation. Based on a nationally representative sample of individuals in the second half of life ( $n=7,837$ individuals aged 40 years and over) in Germany, this recent cross-sectional study [6] showed that daily online social network users (e.g., users of Facebook) reported lower social isolation scores compared with those with less frequent or no social media use. In a sensitivity analysis, this study [6] also examined the association between the use of the internet "for contact with friends and relatives (e.g., e-mail, Facebook, chat, video calls)" and social isolation. Compared to daily users, less frequent users and nonusers had significantly higher social isolation scores. These associations did not differ by sex or educational level. This is in line with a review performed by Leist [7]. She thought that online communities are "places where people can get together and engage in social contact, for example, overcome loneliness at nighttime" [7]. Therefore, she concluded that these sites can help to overcome feelings of loneliness and isolation. In a similar vein, a very recent qualitative study concluded that sites such as Facebook or video chats, via Skype, for example, can help to overcome social isolation among Muslim immigrant older adults in Canada [8]. In sum, the findings are mixed. Moreover, these results come from a time prior to COVID-19. Therefore, they may not directly be transferable to our present time. Thus, future research is urgently required. 


\section{Conclusion}

We would like to conclude with some recommendations for future research. There is a need for further research examining the link between online social network sites/video chat and loneliness as well as social isolation, particularly among the older adults based on nationally representative samples.

While one longitudinal study exists which investigated the link between video chat and depressive symptoms based on a nationally representative sample of older adults [9], there is a complete lack of nationally representative longitudinal studies regarding the link between online social network sites/video chat and loneliness as well as social isolation. Therefore, future studies are urgently required.

Future research is required to elucidate the underlying mechanisms. For example, a recent study [10] among students aged 18-30 years showed that while having positive experiences on social network sites is not associated with social isolation, negative experiences on social network sites is associated with increased levels of social isolation. This suggests that humans weigh negative entities more than positive ones (negativity bias) [10]. Moreover, the intensity of using online social network sites should be investigated among older adults. Even personality factors such as extraversion may play a role in the link between online social network sites/video chat and loneliness or social isolation among older adults.

We truly hope that this commentary will inspire new research in this emerging area. Ultimately, we hope that this knowledge may help to avoid feelings of social isolation and loneliness among older adults dealing with the conditions of the COVID-19 pandemic.

\section{Conflict of Interest Statement}

The authors declare that they have no conflicts of interest.

\section{Funding Sources}

The authors did not receive any funding.

\section{Author Contributions}

A.H.: conceptualization, roles/writing - original draft, and writing - review \& editing. H.H.K.: conceptualization, resources, writing - review \& editing, and supervision.

\section{References}

1 Armitage R, Nellums LB. COVID-19 and the consequences of isolating the elderly. Lancet Public Health. 2020;5(5):e256.

2 Vahia IV, Blazer DG, Smith GS, Karp JF, Steffens DC, Forester BP, et al. COVID-19, mental health and aging: a need for new knowledge to bridge science and service. Am J Geriatr Psychiatry. 2020;28(7):695-7.

3 Klaus D, Engstler H, Mahne K, Wolff JK, Simonson J, Wurm S, et al. Cohort profile: the German Ageing Survey (DEAS). Int J Epidemiol. 2017;46(4):1105-5g.

$4 \mathrm{Yu}$ RP, Mccammon RJ, Ellison NB, Langa KM. The relationships that matter: social network site use and social well-being among older adults in the United States of America. Ageing Soc. 2016;36(9):1826-52.
5 Aarts S, Peek ST, Wouters EJ. The relation between social network site usage and loneliness and mental health in community-dwelling older adults. Int J Geriatr Psychiatry. 2015; 30(9):942-9.

6 Hajek A, König HH. The association between use of online social networks sites and perceived social isolation among individuals in the second half of life: results based on a nationally representative sample in Germany. BMC Public Health. 2019;19(1):40.

7 Leist AK. Social media use of older adults: a mini-review. Gerontology. 2013;59(4):37884.
8 Salma J, Salami B. "Growing Old is not for the Weak of Heart": social isolation and loneliness in Muslim immigrant older adults in Canada. Health Soc Care Community. 2020; 28(2):615-23.

9 Teo AR, Markwardt S, Hinton L. Using Skype to beat the blues: longitudinal data from a national representative sample. Am J Geriatr Psychiatry. 2019;27(3):254-62.

10 Primack BA, Karim SA, Shensa A, Bowman N, Knight J, Sidani JE. Positive and negative experiences on social media and perceived social isolation. Am J Health Promot. 2019; 33(6):859-68. 